\title{
A prospective natural history study of nonoperatively managed Chiari I malformation: does follow-up MRI surveillance alter surgical decision making?
}

\author{
Wesley J. Whitson, MD, ${ }^{1}$ Jessica R. Lane, BA, ${ }^{2}$ David F. Bauer, MD, ${ }^{1}$ and \\ Susan R. Durham, MD, MS² \\ ${ }^{1}$ Section of Neurosurgery, Dartmouth-Hitchcock Medical Center, Lebanon, New Hampshire; and 2Division of Neurosurgery, \\ University of Vermont College of Medicine, Burlington, Vermont
}

\begin{abstract}
OBJECT Chiari malformation Type I (CM-I) in children is a common incidental finding. Resolution of cerebellar tonsil ectopia has been reported, but no studies have followed tonsil position over regular intervals throughout childhood. To better elucidate the clinical and radiological natural history of $\mathrm{CM}$-I in children, the authors prospectively followed up children with nonoperatively managed $\mathrm{CM}-\mathrm{I}$ for up to 7 years.

METHODS The study included all children evaluated for CM-I over a period of 12 years for whom surgery was not initially recommended. The study excluded patients with associated conditions, including syringomyelia and hydrocephalus. For all patients, initial management was nonoperative, and follow-up management consisted of annual cervical spine or brain MRI and clinical examination. At each follow-up examination, the neurological examination findings, subjective symptoms, and the position of the cerebellar tonsils on MR images were recorded. An alteration in tonsillar descent of 2 $\mathrm{mm}$ or greater was considered a change.
\end{abstract}

RESULTS Neurological examination findings did not change over the course of the study in the 52 children who met the inclusion criteria. Although radiological changes were common, no surgeries were performed solely because of radiological change. Overall, tonsil position on radiological images remained stable in $50 \%$ of patients, was reduced in $38 \%$, and increased in $12 \%$. Resolution was seen in $12 \%$ of patients. Radiological changes in tonsil position were seen during every year of follow-up. On average, in any given year, $24 \%$ of images showed some form of change in tonsil position. A total of 3 patients, for whom no changes were seen on MR images, ultimately underwent surgery for subjective clinical reasons.

CONCLUSIONS CM-I in children is not a radiologically static entity but rather is a dynamic one. Radiological changes were seen throughout the 7 years of follow-up. A reduction in tonsillar descent was substantially more common than an increase. Radiological changes did not correlate with neurological examination finding changes, symptom development, or the need for future surgery. Follow-up imaging of asymptomatic children with CM-I did not alter treatment for any patient. It would be reasonable to follow these children with clinical examinations but without regular surveillance MRI.

http://thejns.org/doi/abs/10.3171/2014.12.PEDS14301

KEY WORDS Chiari malformation; natural history; incidental; resolution; MRI; surveillance

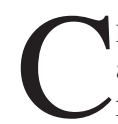
HIARI malformation Type I (CM-I) in children is a common incidental finding. It has been defined radiologically as cerebellar tonsillar descent 5 or more $\mathrm{mm}$ below the plane of the foramen magnum. The true prevalence in the general population is unknown, but two recent retrospective studies reported the incidence among children who underwent MRI to be between $0.4 \%$ and $2.4 \%$. One study examined brain or cervical spine MR images acquired for any reason in over 14,000 children younger than 18 years. ${ }^{17}$ In that cohort, radiological evidence of CM-I was present in $3.6 \%$ of the patients, $68 \%$ of whom were considered asymptomatic at initial evaluation. The overall prevalence of CM-I in this population was $2.4 \%$. Another study included over 5000 brain or cervical spine MR images of patients younger than 20 years. In this group, $1 \%$ of the patients had radiological evidence of CM-I. ${ }^{2}$ When symptomatic patients were excluded, the prevalence of asymptomatic CM-I was $0.4 \%$. 
The natural history for patients with asymptomatic CM-I is poorly understood. A few retrospective case series have outlined the clinical course for these patients, $2,5,10,14,17,21$ but given the varied symptoms attributed to CM-I, determining whether a patient is symptomatic or even whether a patient clinically improves because of operative treatment can be difficult. Confounding matters, retrospective case series describe outcomes in surgically treated versus nonsurgically treated patients rather than symptomatic versus asymptomatic patients because of the difficulty establishing cause-and-effect relationships between the imaging findings and reported symptoms.

Chiari I malformation is often viewed as a static congenital condition, although cases of CM-I acquired secondarily to supratentorial mass effect or excessive spinal fluid drainage from the thecal sac have been reported. ${ }^{8,13,15}$ One series of 124 children with CM-I managed nonoperatively showed no significant change in tonsillar descent. ${ }^{5}$ Multiple case reports have described CM-I patients in whom tonsillar descent decreased or resolved on followup images. $3,4,6,7,9,12,16,20$ Other case reports have described CM-I patients in whom tonsillar descent increased over time. ${ }^{2,10,17}$ In one retrospective case series, the changes in tonsillar descent over time ranged from $-14 \mathrm{~mm}$ to +20 $\mathrm{mm} .^{17}$

To the best of our knowledge, no study to date has prospectively followed the serial MRI patterns of patients with nonoperatively managed CM-I to assess the types of radiological changes seen over time and to determine whether these changes alter clinical management in any way. In this study, to establish recommendations for optimal follow-up imaging, we prospectively followed the clinical and radiological courses of patients with nonoperatively managed CM-I.

\section{Methods}

Study approval was obtained through the Dartmouth Institutional Review Board, and data were collected prospectively for all patients who received a diagnosis of asymptomatic CM-I at the Dartmouth-Hitchcock Medical Center from December 2004 through May 2013. CM-I was defined as tonsillar descent of $5 \mathrm{~mm}$ or more below the foramen magnum as measured on sagittal brain or cervical spine MR images. At the time of initial diagnosis, additional imaging of the entire neural axis was performed to evaluate for associated pathology such as a supratentorial mass lesion, syringomyelia, or a tethered spinal cord. At the surgeon's discretion, patients were offered operative decompression, typically based on the presence of syringomyelia, occipital Valsalva maneuver-induced headaches, or brainstem compression symptoms attributed to the CM-I. A discussion of surgical decision making for CM-I is beyond the scope of this paper. If surgical intervention was not initially recommended, the patient was managed nonoperatively and was included in this natural history study.

Patients were excluded from study participation if their initial evaluation revealed a history of hydrocephalus, spinal dysraphism, syringomyelia, craniosynostosis, achondroplasia, or chromosomal abnormalities. A syrinx was defined as dilation of the central canal of the spinal cord of $3 \mathrm{~mm}$ or more.

Patients were scheduled for routine follow-up evaluations annually for 5 years and then again at 7 years after initial diagnosis. Not all patients completed follow-up visits as scheduled, but all available data were included in the study. We included any patient who had at least 1 follow-up clinic visit in which imaging was performed. Some patients missed appointments sporadically, and others were entirely lost to follow-up. At each appointment, the patient history was updated, a neurological examination was performed, and brain or cervical spine MRI was performed. For each follow-up imaging study, tonsil descent was independently measured by a neuroradiologist and a pediatric neurosurgeon. Any discrepancies in measurement were resolved with a final review by an additional neurosurgeon. At each follow-up interval, patients were assessed for reduced, increased, or stable measured radiological tonsillar descent compared with that found on all prior images, including the baseline images and any subsequent images obtained before the follow-up appointment. We considered a change to be an alteration in the degree of tonsillar descent of $2 \mathrm{~mm}$ from that found on baseline or any prior study. We considered resolution of the CM-I to be tonsil descent that decreased to less than $5 \mathrm{~mm}$.

\section{Results}

Over the 12-year study period, 228 patients at our clinic had CM-I. Of these, 145 (64\%) patients were managed operatively and $83(36 \%)$ were managed nonoperatively. Among the nonoperatively managed patients, 55 met inclusion criteria for this study. Of those who did not meet inclusion criteria, 14 were lost to follow-up, 13 had hydrocephalus, 3 had achondroplasia, and 1 had a chromosomal abnormality. Because 3 patients elected to proceed with surgical intervention over the course of the study, they became ineligible to complete the natural history follow-up study. Thus, a total of 52 patients were included in the natural history analysis. At the time of initial evaluation, patient ages ranged from 2 to 16 years (mean age 8.2 years), and 27 patients were girls and 25 were boys (Table 1).

Over the course of the study, no change in objective neurological examination findings was found for any pa-

TABLE 1. Characteristics of study patients with CM-I

\begin{tabular}{lcc}
\hline Characteristic & $\begin{array}{c}\text { Did Not Undergo } \\
\text { Surgery }\end{array}$ & $\begin{array}{c}\text { Dropped Out of Study } \\
\text { to Undergo Surgery }\end{array}$ \\
\hline Age (years) & & \\
\hline Mean at presentation & 8.2 & 10 \\
\hline Range at presentation & $2-16$ & $1-16$ \\
\hline Sex, no. (\%) & & $2(67)$ \\
\hline M & $25(48)$ & $1(33)$ \\
\hline Tonsillar descent (mm) & $27(52)$ & 14.3 \\
\hline Initial mean & 11 & $13-15$ \\
\hline Initial range & $5-26$ & 0 \\
\hline Range of change & -19 to +7 & \\
\hline
\end{tabular}


tient. Two patients reported development of exertional headaches and dropped out of the study to undergo surgery for this reason. One patient experienced speech delay and irritability and also dropped out of the study to undergo surgery. No symptoms concerning for brainstem compression developed in any patient.

The initial images that prompted referral to our clinic were obtained for a variety of reasons: 16 for headache, 11 for persistent emesis, 5 for trauma, 5 for endocrine abnormalities, 4 for seizures, 4 for movement disorders, 2 for visual changes, 2 for scoliosis, 1 for incontinence, 1 for behavioral changes, and 1 for a fatty neck mass. For all patients, the referring providers consulted pediatric neurosurgery to complete the evaluation for a new diagnosis of CM-I and to determine whether surgery might be indicated. For all included patients, the symptoms were considered unlikely to be associated with the CM-I. For patients in whom headache was the presenting symptom, the headache did not have the exertional or Valsalva maneuver-induced component typical of a CM-I headache.

Initial tonsillar descent ranged from 5 to $26 \mathrm{~mm}$ (mean $11 \mathrm{~mm}$ ) (Fig. 1). Figure 2 shows some examples of tonsillar descent at the initial visit. Over the entire course of the study, tonsillar descent remained stable in 26 patients $(50 \%)$ and changed more than $2 \mathrm{~mm}$ in 26 patients (50\%). Of the 26 in whom change was demonstrated, tonsillar descent was reduced in $20(77 \%)$ and increased in 6 (23\%). CM-I completely resolved over the course of the study in 6 patients $(12 \%)$. Changes in tonsillar descent ranged from -19 to $+7 \mathrm{~mm}$. The annual average change in millimeters of descent is shown in Fig. 3. MRI data regarding changes in tonsillar descent by study year are summarized in Table 2.

Changes were seen during every year of follow-up. In any given year, radiological changes were seen for $14 \%-$ $32 \%$ of patients. On average, $24 \%$ of images from any given year showed some kind of change in tonsillar descent; reduction was seen for an average of $19 \%$ of patients and an increase for an average of 5\% of patients (Fig. 4). The mean absolute value of change in any given year was $4.6 \mathrm{~mm}$. The percentage of patients in whom a change occurred was highest at year 2 and lowest at year 7 of followup. Findings for other years were relatively similar. The annual total percentage change is shown in Fig. 5.

Complete resolution of CM-I was seen at various times throughout follow-up. Age at presentation ranged from 2

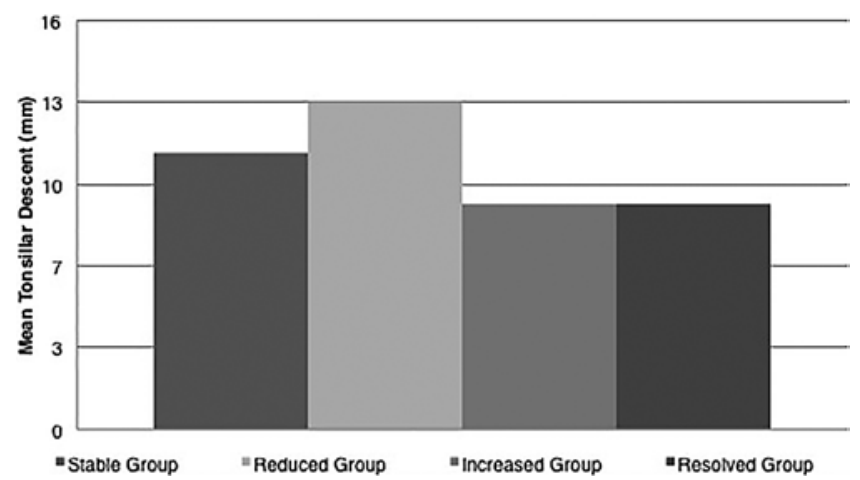

FIG. 1. Mean cerebellar tonsillar descent at initial evaluation.
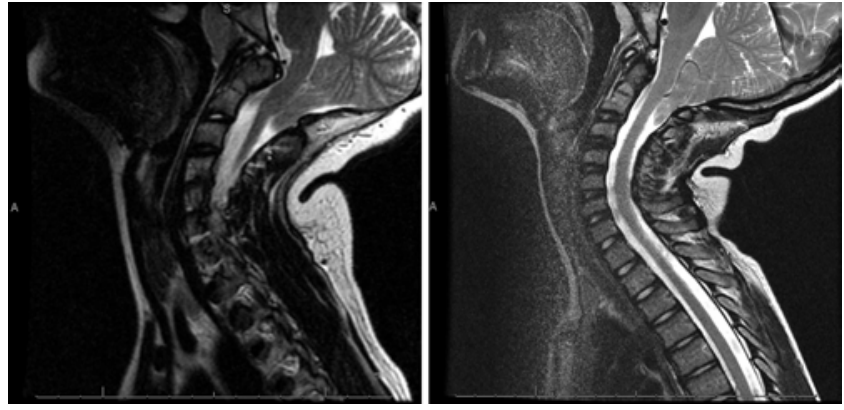

FIG. 2. Sagittal MR images showing significant cerebellar tonsillar descent in 2 asymptomatic patients at initial evaluation.

to 14 years (mean age 6 years). Initial tonsillar descent in this subgroup ranged from 6 to $19 \mathrm{~mm}$ (mean descent 9 $\mathrm{mm}$ ). The average time from initial evaluation to resolution was 2.8 years. The patient for whom time to resolution was longest, 7 years, was the oldest in this group and at initial evaluation was 14 years of age. The rest of the patients in this group were 2-7 years of age. The time courses of the various types of change can be seen in Figs. 6-8. Age at initial evaluation, sex, and initial tonsillar descent were analyzed to determine if they were predictive of CM-I resolution. None was statistically significant as a predictor of CM-I resolution. Figure 9 shows examples of images obtained initially and again at the time of resolution.

For no patients in our series did a syrinx develop during the study period. A syrinx is defined as a 3-mm or greater dilation of the central canal of the spinal cord.

For 2 patients in our series, a fatty filum terminale with a normally positioned conus medullaris at the level of L1-2 was found. Neither patient underwent filum lysis. For both patients, the extent of tonsillar descent decreased over time and each remained clinically stable without tethered cord symptoms.

Initial management was nonoperative for 3 patients in our series, but surgical decompression was performed later. For these patients, no radiological changes in CM-I were seen, and all remained neurologically intact. Of the surgeries that were performed, all were for subjective clinical symptoms and none were for brainstem compression. For 1 patient, 1 year of age at initial evaluation, tonsillar descent was $15 \mathrm{~mm}$. Surgery was performed at age 3 for speech delay and irritability, but the boy was too young to

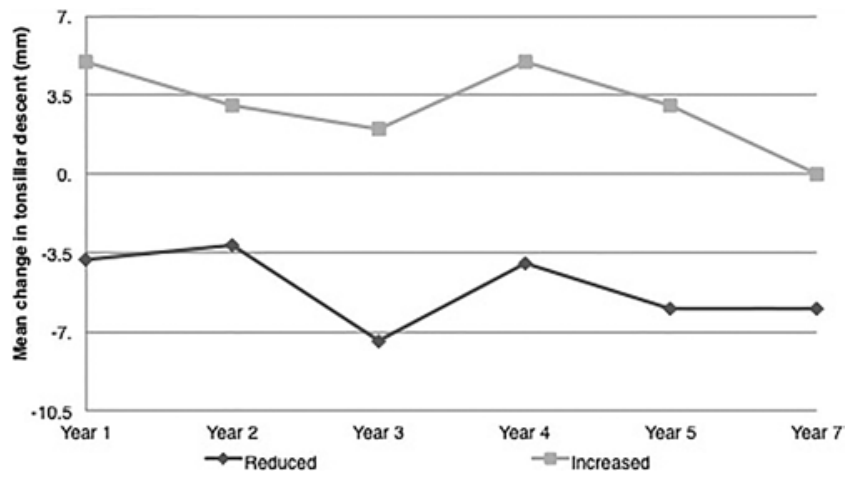

FIG. 3. Mean change of cerebellar tonsillar descent per year. Evaluations were not routinely performed during Year 6 . 
TABLE 2. Annual MRI results over the study period*

\begin{tabular}{lcccccc}
\hline \multicolumn{1}{c}{ Result } & Year 1 & Year 2 & Year 3 & Year 4 & Year 5 & Year 7 \\
\hline Total MRI studies (no.) & 39 & 22 & 23 & 15 & 13 & 7 \\
\hline Total changed (no.) & 8 & 7 & 7 & 4 & 3 & 1 \\
\hline Cerebellar tonsils & & & & & & \\
\hline Reduced size (no.) & 6 & 6 & 6 & 3 & 2 & 1 \\
\hline Reduction in mm/patient & $2 / 2 / 4 / 5 / 5 / 5$ & $2 / 2 / 2 / 2 / 4 / 7$ & $3 / 6 / 7 / 8 / 13$ & $2 / 2 / 8$ & $2 / 10$ & 6 \\
\hline Increased size (no.) & 2 & 1 & 1 & 1 & 1 & 0 \\
\hline Increase in mm/patient & $3 / 7$ & 3 & 2 & 5 & 3 & 0 \\
\hline Resolved (no.) & 1 & 3 & 1 & 0 & 0 & 1 \\
\hline
\end{tabular}

* Evaluations were not routinely performed during Year 6 .

assess for the presence of an exertional headache. These symptoms did not improve postoperatively. For another patient, 13 years of age, initial tonsillar descent was 13 $\mathrm{mm}$; this patient opted for surgery 5 years later because of exertional headaches, which did decrease postoperatively. For the third patient, 16 years of age, tonsillar descent was $15 \mathrm{~mm}$; this patient also chose surgery because of exertional headaches, which did not decrease postoperatively. Figure 10 shows the images of a patient who underwent surgery, taken at initial evaluation and before surgery.

\section{Discussion}

CM-I in children can radiologically change over time. In our series, the degree of tonsillar descent changed in $50 \%$ of children managed nonoperatively. On average, nearly $25 \%$ of surveillance scans done annually showed a change in tonsillar descent of $2 \mathrm{~mm}$ or more from the initial or prior measurement. On average, a change was shown in any given year for $24 \%$ of patients. Significant changes in tonsillar descent and even resolution of CM-I were seen as far out as the seventh study year (the final year of this study). Presumably, changes might be seen even beyond this time frame. In each year of follow-up, more patients showed reduction in tonsillar descent than increase.

The literature contains multiple reports of radiologically observed reduction and resolution of CM-I. The 78 cases with available patient demographics (rather than large series without details on specific patients) are sum-

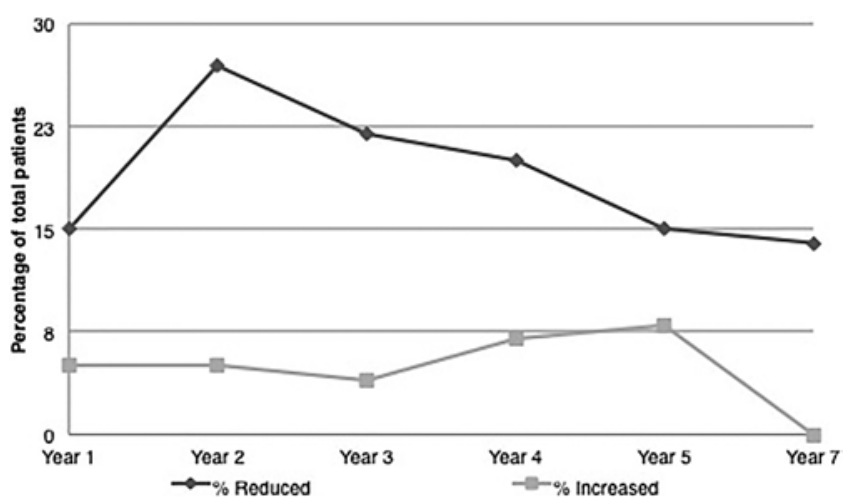

FIG. 4. Percentage of patients in each year of follow-up for whom the degree of cerebellar tonsillar descent increased or decreased. Evaluations were not routinely performed during Year 6 . marized in Table 3. Patient ages range from 1 to 16 years (mean age 7 years), and $60 \%$ of patients were male. Initial tonsillar descent is not reported in many of the articles, but range of descent was 7-13 $\mathrm{mm}$ (mean $10 \mathrm{~mm}$ ). Follow-up times ranged from 2 to 11 years (mean 5 years). For these 78 patients, tonsil herniation increased for 10 , reduced for 51, and resolved for 17. Publication bias may explain the preponderance of reduction documented in the literature; 6 of the 10 patients for whom a CM-I increase was reported participated in a single natural history series. ${ }^{17}$ The degree of change in tonsillar descent was rarely reported, so we were unable to analyze this factor.

Of clinical relevance are those patients in whom CM-I completely resolved during the course of the study. In our series, in no patients did CM-I completely resolve and then reappear on subsequent images. Our study demonstrates that CM-I will show radiological resolution over time in as many as $12 \%$ of asymptomatic children. No statistically significant factors to predict radiological resolution were identified in our study.

Surgical decision making for patients with CM-I can be challenging because the symptoms can be difficult to definitively attribute to the malformation. In our series, no patient in whom a change in tonsillar descent was seen experienced symptoms leading to a decision to perform surgery. The abnormalities seen on images for the 3 study patients who underwent surgery remained stable at the time of the decision for surgery. Surgery was performed on the basis of symptoms and not surveillance imaging

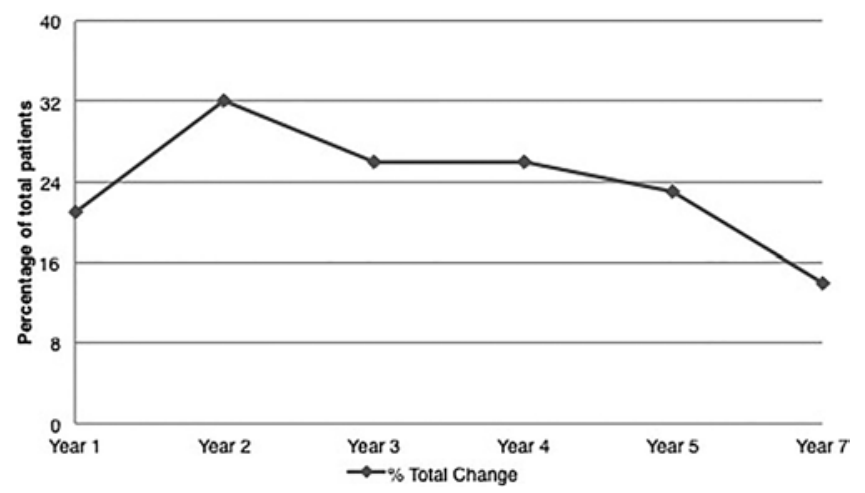

FIG. 5. Percentage of patients in each year of follow-up for whom the degree of cerebellar tonsillar descent showed any type of change (reduction or increase). Evaluations were not routinely performed during Year 6. 


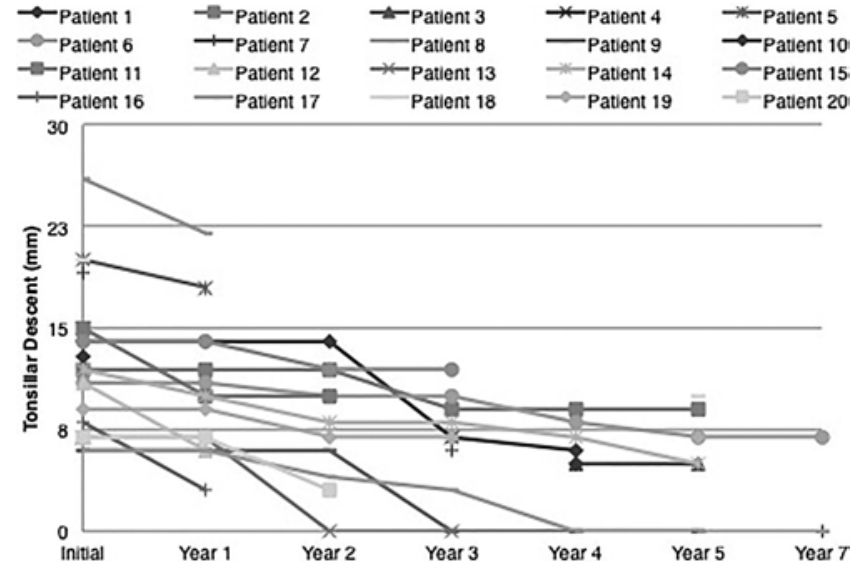

FIG. 6. Tonsillar descent over time for patients with radiological evidence of reduced $\mathrm{CM}-\mathrm{I}$. Evaluations were not routinely performed during Year 6.

findings. Two patients who underwent surgery experienced a change in headache quality with a new exertional component. For one of these patients, headaches decreased after surgery and the improvement was sustained at last follow-up evaluation 1 year postoperatively. Another of these patients experienced persistent headaches and additional postoperative pain and returned to the emergency department multiple times for assessment of these headaches. The third patient was a 3-year-old boy who initially underwent MRI for speech delay. He displayed irritability but was too young for ascertainment of the presence of exertional headaches. Suboccipital decompression was performed, but the speech delay and irritability did not improve.

Other published retrospective natural history studies of CM-I have reported a similar small percentage of patients who initially underwent nonoperative management but ultimately underwent surgery. Novegno et al. reported surgery having been performed for 1 (4.5\%) of 22 patients; this patient had experienced worsening ventriculomegaly and tonsillar descent. ${ }^{14}$ Massimi et al. reported clinical and radiological worsening of CM-I for 2 of 16 patients with progressive hydrocephalus. ${ }^{10}$ These 2 patients underwent successful endoscopic third ventriculostomy without suboccipital decompression. Benglis et al. reported delayed

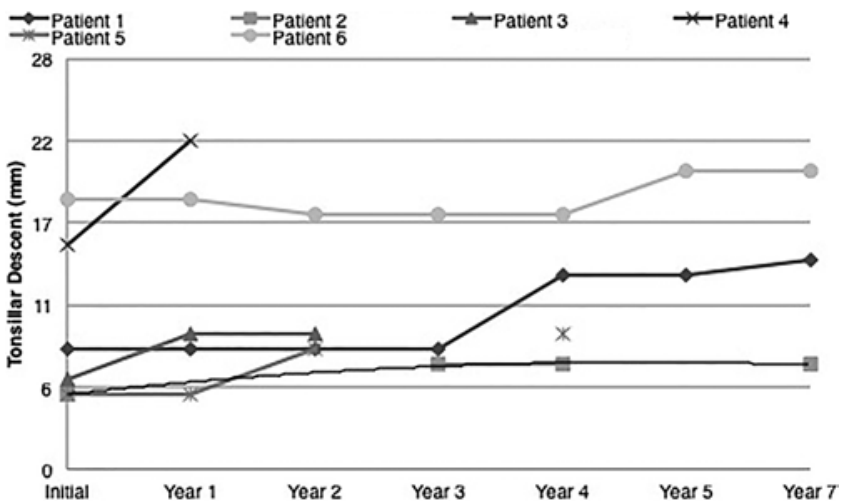

FIG. 7. Tonsillar descent over time for patients with radiological evidence of increased $\mathrm{CM}-\mathrm{I}$. Evaluations were not routinely performed during Year 6.

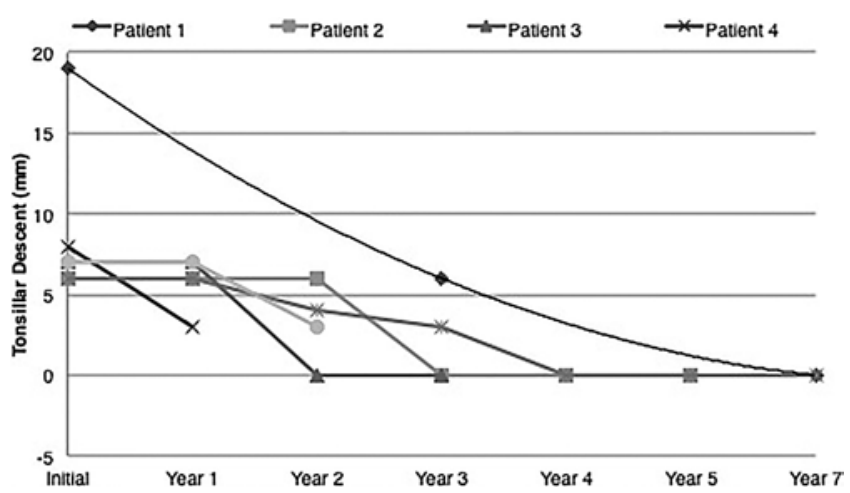

FIG. 8. Tonsillar descent over time for patients with radiological evidence of resolved CM-I. Evaluations were not routinely performed during Year 6.

surgery for 5 (4\%) of 124 patients with CM-I, ${ }^{5}$ and Strahle et al. reported delayed surgery for $14(9.5 \%)$ of 147 patients. ${ }^{17}$ In our series, $3(5.5 \%)$ of 55 patients underwent delayed suboccipital decompression. Because patients with hydrocephalus were excluded from our study, we could not evaluate associations between worsening hydrocephalus and CM-I; comparison of delayed surgery rates between our study and the others mentioned here would be unreliable. The results of our study should not be extrapolated to include patients with hydrocephalus, in whom CM-I can follow a different clinical course.

The literature contains reports of patients with CM-I who were initially symptomatic but improved over time. Novegno et al. described 3 patients with symptomatic CM-I for whom radiological and clinical improvement was later seen after nonoperative management. ${ }^{14}$ Although we found no correlation between radiological changes and symptom changes, we did not follow the natural history of symptomatic patients who underwent nonoperative management.

Given the high prevalence of asymptomatic tonsillar descent among children, as well as the variations in tonsillar descent among asymptomatic patients that are consistently reported in the literature, the term Chiari I "malformation" may not be appropriate because it implies a pathological state. Most of the children in our study remained asymptomatic throughout the study period of up to 7 years. For these asymptomatic patients, a term such as tonsil ectopia or Chiari I anomaly might more appropriately describe an imaging finding that is not necessarily
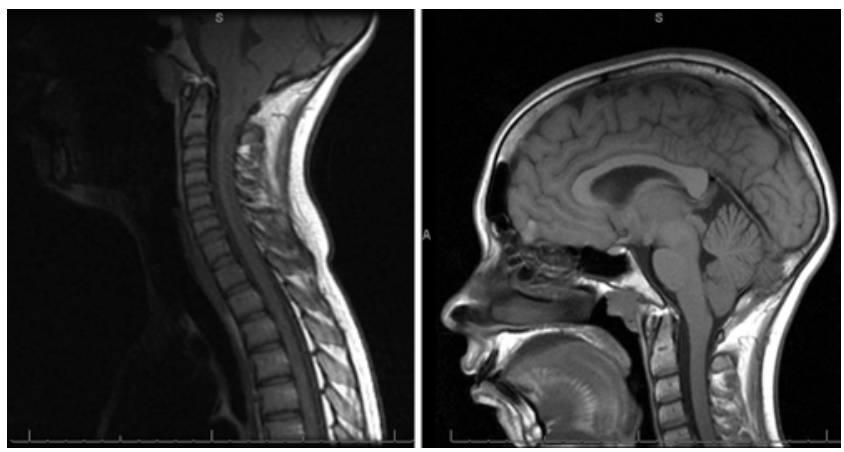

FIG. 9. Sagittal MR images of a patient at initial evaluation (left) and at the time of spontaneous resolution (right) of a $\mathrm{CM}-\mathrm{I}$. 

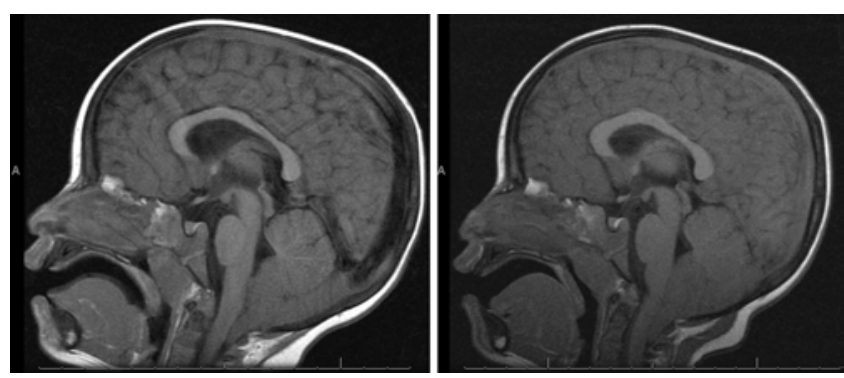

FIG. 10. Sagittal MR images of a patient at initial evaluation (left) and before surgery (right), showing no measurable change in tonsillar descent.

pathological. An analogy can be made to the difference between ventriculomegaly and hydrocephalus: ventriculomegaly does not necessarily imply a pathological con- dition, but hydrocephalus does. "Chiari I malformation" could then more specifically refer to symptomatic patients with a clinical, not simply a radiological, syndrome.

A finding of questionable significance (because of the small sample size) is the presence of fatty filum terminale and normal position of the conus medullaris in 2 patients with CM-I. For both patients, the degree of tonsillar descent decreased over time and initial tonsillar descent was $12 \mathrm{~mm}$. By 5 years of follow-up, descent had decreased to $9 \mathrm{~mm}$ for 1 patient and to $5 \mathrm{~mm}$ for the other. Although reports have stated that tethered spinal cord might cause CM-I and lead to increased tonsillar descent, ${ }^{1}$ our findings do not support this hypothesis. The fatty film did not lead to increased tonsillar descent and did not even prevent reduction in tonsil herniation.

Our study did not address nonoperative management

TABLE 3. Review of cases of change in tonsillar descent reported in English-language literature

\begin{tabular}{|c|c|c|c|c|c|}
\hline Authors \& Year & $\begin{array}{l}\text { Patient Age } \\
\text { (years) }\end{array}$ & Patient Sex & $\begin{array}{l}\text { Initial Tonsillar } \\
\text { Descent (mm) }\end{array}$ & $\begin{array}{c}\text { Follow-Up } \\
\text { (years) }\end{array}$ & Type of Change \\
\hline Sudo et al., 1990 & 14 & M & - & 2 & Resolved \\
\hline Castillo \& Wilson, 1995 & 9 & $\mathrm{~F}$ & - & 4 & Resolved \\
\hline Avellino et al., 1996 & 5 & M & - & 5 & Reduced \\
\hline Avellino et al., 1999 & 5 & $\mathrm{~F}$ & - & 11 & Reduced \\
\hline Sun et al., 2000 & 11 & M & - & 1 & Increased after resolution \\
\hline Rafia \& Pascual-Castroviejo, 2001 & 4 & M & - & 4 & Reduced \\
\hline Sun et al., 2001 & 7 & M & 13 & 2 & Resolved \\
\hline \multirow[t]{14}{*}{ Tokunaga et al., 2001} & 5 & $\mathrm{~F}$ & - & 2 & Reduced \\
\hline & 6 & M & - & 9 & Reduced \\
\hline & 7 & M & - & 5 & Resolved \\
\hline & 7 & M & - & 6 & Reduced \\
\hline & 8 & M & - & 8 & Reduced \\
\hline & 10 & M & - & 3 & Reduced \\
\hline & 10 & M & - & 9 & Reduced \\
\hline & 10 & $\mathrm{~F}$ & - & 2 & Reduced \\
\hline & 10 & M & - & 8 & Resolved \\
\hline & 11 & M & - & 4 & Reduced \\
\hline & 13 & $\mathrm{~F}$ & - & 2 & Reduced \\
\hline & 14 & $\mathrm{~F}$ & - & 8 & Reduced \\
\hline & 15 & $\mathrm{~F}$ & - & 4 & Reduced \\
\hline & 16 & $\mathrm{~F}$ & - & 2 & Resolved \\
\hline Guillen \& Costa, 2004 & 6 & $\mathrm{~F}$ & - & 8 & Resolved \\
\hline Jatavallabhula et al., 2006 & 1 & M & - & 4 & Resolved \\
\hline \multirow[t]{2}{*}{ Miller et al., 2008} & 1 & $\mathrm{~F}$ & 12 & 4 & Reduced \\
\hline & 1 & $\mathrm{~F}$ & 10 & 4 & Resolved \\
\hline \multirow[t]{6}{*}{ Novegno et al., 2008} & 2 & M & - & 10 & Resolved \\
\hline & 2 & M & - & 9 & Reduced \\
\hline & 2 & M & - & 5 & Increased \\
\hline & 4 & M & - & 5 & Reduced \\
\hline & 5 & $\mathrm{~F}$ & - & 6 & Reduced \\
\hline & 6 & $\mathrm{~F}$ & - & 3 & Increased \\
\hline \multirow[t]{2}{*}{ Massimi et al., 2011} & 2 & M & 7 & 7 & Increased \\
\hline & 2 & M & 8 & 11 & Reduced \\
\hline
\end{tabular}

$-=$ not reported. 
for patients with CM-I and an associated syrinx because patients with a syrinx were excluded and no syrinx developed de novo in any patient during the study period. Multiple reports in the literature describe reduction or resolution of a syrinx with reduction or resolution of a nonoperatively managed CM-I.3,4,7,18,20,21 Another report describes a patient in whom a syrinx associated with a CM-I increased and was managed nonoperatively. ${ }^{14}$ Because our study contained no patients with a syrinx, we do not have data on which to base treatment recommendations for patients with asymptomatic CM-I and a syrinx.

Last, the idea of cerebellar tonsil ascent with age as part of normal development has been proposed. ${ }^{11}$ In their retrospective natural history study, Strahle et al. found a higher rate of improvement among patients 12-18 years of age than among younger patients. ${ }^{17}$ In our series, age was not a statistically significant factor for any type of change in the degree of tonsillar descent. Although the idea of patients "growing into their larger skulls" resulting in less tonsillar descent makes intuitive sense, we found no evidence of this phenomenon in our study.

The cause of the observed natural variation in tonsillar descent among these patients remains unclear. Future studies could investigate whether such things as intubation and its associated changes in ventilatory dynamics, anesthetic agents, patient positioning, or hydration affect the position of the cerebellar tonsils, accounting for the position changes of the tonsils seen over time.

The main clinical question arising from this study is whether asymptomatic patients with nonoperatively managed CM-I should undergo regular follow-up MRI. From a basic clinical perspective, surgery for CM-I without associated hydrocephalus or syrinx is entirely based on symptoms and neurological examination findings. The presence of a CM-I or an increase in tonsillar descent is not in itself an indication for surgery. Because of the frequency with which asymptomatic CM-I is found, clinical evaluation and judgment, rather than merely radiological findings, remain the standard for surgical intervention for any patient with a CM-I. Over the course of this study, only a small percentage of patients who initially underwent nonoperative management went on to undergo suboccipital decompression. None of these decisions were based on imaging findings. Rather, they were based on clinical changes. No patient exhibited neurological deterioration, development of syringomyelia, or showed any signs of brainstem compression over the entire follow-up period. On the basis of these data, regular surveillance MRI may not be necessary.

Regular clinical follow-up for asymptomatic patients with CM-I including a history of symptoms and neurological examination may still be useful. No patient in this series demonstrated an objective change in neurological examination findings. However, in 1 patient, exertional headaches developed but resolved after subsequent surgery. Although this patient accounts for less than $2 \%$ of the 52 patients initially involved in the study, a clinically beneficial result was obtained. Should symptoms of brainstem compression, syringomyelia, or exertional headaches develop, MR images could be obtained at that time to help with surgical decision making. Although for no patients in our study did a syrinx develop over the study period, others have reported the occurrence of this phenomenon..$^{17,19}$ For this reason, we believe that clinical surveillance follow-up with imaging performed only on the basis of new symptom development remains valuable.

Clinical judgment is still involved in determining the course of follow-up for patients with asymptomatic CM-I. We believe that for most patients, initial consultation with a follow-up clinic appointment a year later is sufficient. We recommend repeat imaging solely on the basis of new or changing symptoms or examination findings. For young children or children without the capacity to communicate worsening symptoms, we recommend follow-up for a longer time, potentially with repeat imaging, to avoid missing a change in this vulnerable population.

This study has several limitations. First, there is selection bias at initial presentation for surgical versus nonsurgical management. Our goal with this paper was to describe the natural history for patients who did not undergo surgery; a thorough discussion of the indications for surgical intervention for CM-I is beyond the scope of this paper. A valuable follow-up study would evaluate symptomatic patients who initially underwent nonoperative management to prospectively track symptomatic, clinical, and radiological changes over time. Additionally, although we started with a total of 52 patients, follow-up data at 7 years were available for only 7 patients. These numbers clearly limit the value of statistical analysis at year 7. However, the observation that tonsillar descent continues to change this far out in follow-up, and that these changes do not affect surgical decision making, remains valid. Our study was also limited because the number of nonoperatively managed patients was low enough that obtaining statistical significance for any factors predictive of change was unlikely. A larger cohort would have provided adequate power to establish significance for some of the variables we analyzed or to verify the null hypothesis. Last, the minimal crossover to surgery in our study makes it difficult to compare findings for patients in the crossover group with findings for patients who continued with nonoperative management throughout the study period.

\section{Conclusions}

For many children with CM-I, the degree of cerebellar tonsillar descent changes over time. Radiological changes in cerebellar tonsillar descent were seen throughout the 7-year study period. For some patients, tonsillar descent increased over time, but for others, it decreased or fully resolved. Radiological change did not correlate with symptom development or the need for surgery. Symptoms developed during the study period for only a few patients with nonoperatively managed CM-I. Regular clinical followup evaluations to assess the development of symptoms is valuable, but regular surveillance MRI does not seem to contribute to the clinical management of these patients.

\section{Acknowledgment}

We acknowledge Jason D. Balkman, MD, a radiologist at our institution who assisted with image selection and processing for this paper. 


\section{References}

1. Abel TJ, Chowdhary A, Gabikian P, Ellenbogen RG, Avellino AM: Acquired Chiari malformation Type I associated with a fatty terminal filum. Case report. J Neurosurg 105 (4 Suppl):329-332, 2006

2. Aitken LA, Lindan CE, Sidney S, Gupta N, Barkovich AJ, Sorel M, et al: Chiari type I malformation in a pediatric population. Pediatr Neurol 40:449-454, 2009

3. Avellino AM, Britz GW, McDowell JR, Shaw DW, Ellenbogen RG, Roberts TS: Spontaneous resolution of a cervicothoracic syrinx in a child. Case report and review of the literature. Pediatr Neurosurg 30:43-46, 1999

4. Avellino AM, Kim DK, Weinberger E, Roberts TS: Resolution of spinal syringes and Chiari I malformation in a child. Case illustration. J Neurosurg 84:708, 1996

5. Benglis D Jr, Covington D, Bhatia R, Bhatia S, Elhammady MS, Ragheb J, et al: Outcomes in pediatric patients with Chiari malformation Type I followed up without surgery. J Neurosurg Pediatr 7:375-379, 2011

6. Castillo M, Wilson JD: Spontaneous resolution of a Chiari I malformation: MR demonstration. AJNR Am J Neuroradiol 16:1158-1160, 1995

7. Guillen A, Costa JM: Spontaneous resolution of a Chiari I malformation associated syringomyelia in one child. Acta Neurochir (Wien) 146:187-191, 2004

8. Hatae R, Kohri R, Maeda K, Miyazono M: A rare case of Chiari type-1 malformation accompanied by symptomatic cerebrospinal fluid hypovolemia: comparison of congenital Chiari type-1 malformation and acquired Chiari malformation secondary to cerebrospinal fluid hypovolemia: case report. Neurol Med Chir (Tokyo) 54:558-562, 2014

9. Jatavallabhula NS, Armstrong J, Sgouros S, Whitehouse W: Spontaneous resolution of isolated Chiari I malformation. Childs Nerv Syst 22:201-203, 2006

10. Massimi L, Caldarelli M, Frassanito P, Di Rocco C: Natural history of Chiari type I malformation in children. Neurol Sci 32 (Suppl 3):S275-S277, 2011

11. Mikulis DJ, Diaz O, Egglin TK, Sanchez R: Variance of the position of the cerebellar tonsils with age: preliminary report. Radiology 183:725-728, 1992

12. Miller JH, Limbrick DD, Callen M, Smyth MD: Spontaneous resolution of Chiari malformation Type I in monozygotic twins. J Neurosurg Pediatr 2:317-319, 2008

13. Mokri B: Spontaneous CSF leaks: low CSF volume syndromes. Neurol Clin 32:397-422, 2014

14. Novegno F, Caldarelli M, Massa A, Chieffo D, Massimi L, Pettorini B, et al: The natural history of the Chiari Type I anomaly. J Neurosurg Pediatr 2:179-187, 2008
15. Peleggi AF, Lovely TJ: Treatment of delayed Chiari malformation and syringomyelia after lumboperitoneal shunt placement: Case report and treatment recommendations. Surg Neurol Int 3:101, 2012

16. Rafia S, Pascual-Castroviejo I: [Syringohydromyelia. Report of a case which resolved spontaneously.] Rev Neurol 32:635-637, 2001 (Span)

17. Strahle J, Muraszko KM, Kapurch J, Bapuraj JR, Garton HJ, Maher CO: Natural history of Chiari malformation Type I following decision for conservative treatment. J Neurosurg Pediatr 8:214-221, 2011

18. Sudo K, Doi S, Maruo Y, Tashiro K, Terae S, Miyasaka K, et al: Syringomyelia with spontaneous resolution. J Neurol Neurosurg Psychiatry 53:437-438, 1990

19. Sun JC, Steinbok P, Cochrane DD: Spontaneous resolution and recurrence of a Chiari I malformation and associated syringomyelia. Case report. J Neurosurg 92 (2 Suppl):207-210, 2000

20. Sun PP, Harrop J, Sutton LN, Younkin D: Complete spontaneous resolution of childhood Chiari I malformation and associated syringomyelia. Pediatrics 107:182-184, 2001

21. Tokunaga M, Minami S, Isobe $\mathrm{K}$, Moriya $\mathrm{H}$, Kitahara $\mathrm{H}$, Nakata Y: Natural history of scoliosis in children with syringomyelia. J Bone Joint Surg Br 83:371-376, 2001

\section{Author Contributions}

Conception and design: Whitson, Bauer, Durham. Acquisition of data: Whitson, Lane, Durham. Analysis and interpretation of data: Whitson, Bauer, Durham. Drafting the article: Whitson. Critically revising the article: Whitson, Bauer, Durham. Reviewed submitted version of manuscript: all authors. Approved the final version of the manuscript on behalf of all authors: Whitson. Statistical analysis: Whitson. Administrative/technical/material support: Whitson, Bauer, Durham. Study supervision: Bauer, Durham.

\section{Supplemental Information}

\section{Previous Presentation}

Presented at the Annual Meeting of the Congress of Neurological Surgeons, Boston, Massachusetts, October 18-22, 2014.

\section{Correspondence}

Wesley J. Whitson, Pediatric Neurosurgery, Dartmouth-Hitchcock Medical Center, One Medical Center Dr., Lebanon, NH 03756. email: wesley.j.whitson@hitchcock.org. 